\title{
In-situ multiscale shear failure of a bistable composite tape-spring
}

\author{
Bing Wang ${ }^{1,2^{*}}$, Keith A Seffen ${ }^{1}$, Simon D Guest ${ }^{1}$, Tung-Lik Lee ${ }^{3}$, \\ Shi Huang ${ }^{4}$, Shifeng Luo ${ }^{4}$, Jiawei Mi ${ }^{4,5}$ \\ ${ }^{1}$ Department of Engineering, University of Cambridge, Cambridge, CB2 1PZ, UK \\ ${ }^{2}$ School of Mechanical Engineering and Automation, Fuzhou University, Fuzhou, \\ 350108, China \\ ${ }^{3}$ ISIS Neutron and Muon Source, Rutherford Appleton Laboratory, Harwell Oxford, \\ Didcot, OX11 0QX, UK \\ ${ }^{4}$ Department of Engineering, University of Hull, Hull, HU6 7RX, UK \\ ${ }^{5}$ School of Materials Science and Engineering, Shanghai Jiao Tong University, \\ Shanghai, 200240, China \\ * Corresponding to: bw407@ cam.ac.uk (B Wang)
}

\begin{abstract}
:
A bistable composite tape-spring (CTS) is stable in both the extended and coiled configurations, with fibres oriented at $\pm 45^{\circ}$. It is light weight and multifunctional, and has attracted growing interest in shape-adaptive and energy harvesting systems in defence-, civil- and, especially aerospace engineering. The factors governing its bistability have been well-understood, but there is limited research concerning the mechanics of structural failure: here, we investigate the shear failure mechanisms in particular. We perform in-situ neutron diffraction on composite specimens using the ENGIN-X neutron diffractometer at Rutherford Appleton Laboratory (STFC, UK), and shear failure is characterised at both macroscopic and microscopic scales. Elastic and viscoelastic strain evolutions at different strain levels reveal the fundamentals of micromechanical shear failure, and their temperature dependency. Multiscale shear failure mechanisms are then proposed, which will benefit the optimisation of structural design to maintain structural integrity of CTS in aerospace applications.
\end{abstract}

\section{Keywords:}

Composite; tape-spring; elasticity and viscoelasticity; neutron diffraction; failure mechanism. 
A bistable composite tape-spring (CTS) is stable in both the extended and coiled configurations, see Figures 1 (a) and (b), and has been used in defence-, civil- and aerospace applications. Glass fibre reinforced polypropylene (GF/PP) composite is a well-established material system for fabricating bistable CTS structures, which was discovered by Daton-Lovett in 1996 [1], and consolidated theoretically by Cambridge researchers [2-5]. The factors governing its bistability are well-understood: it is a combination of material constitutive behaviour, initial geometrical proportions, and a geometrically non-linear response during deformation [5]. This inherent structural behaviour has similarities with the lock-stay or side-stay assemblies within an aircraft landing gear: these extend and retract with the gear but remain in a fixed position when the gear is stowed in the bay or locked in the down position. Thus, it is envisaged that by using CTS structures, weight, complexity and maintenance could be further reduced compared to the conventional lock-link assemblies [6].
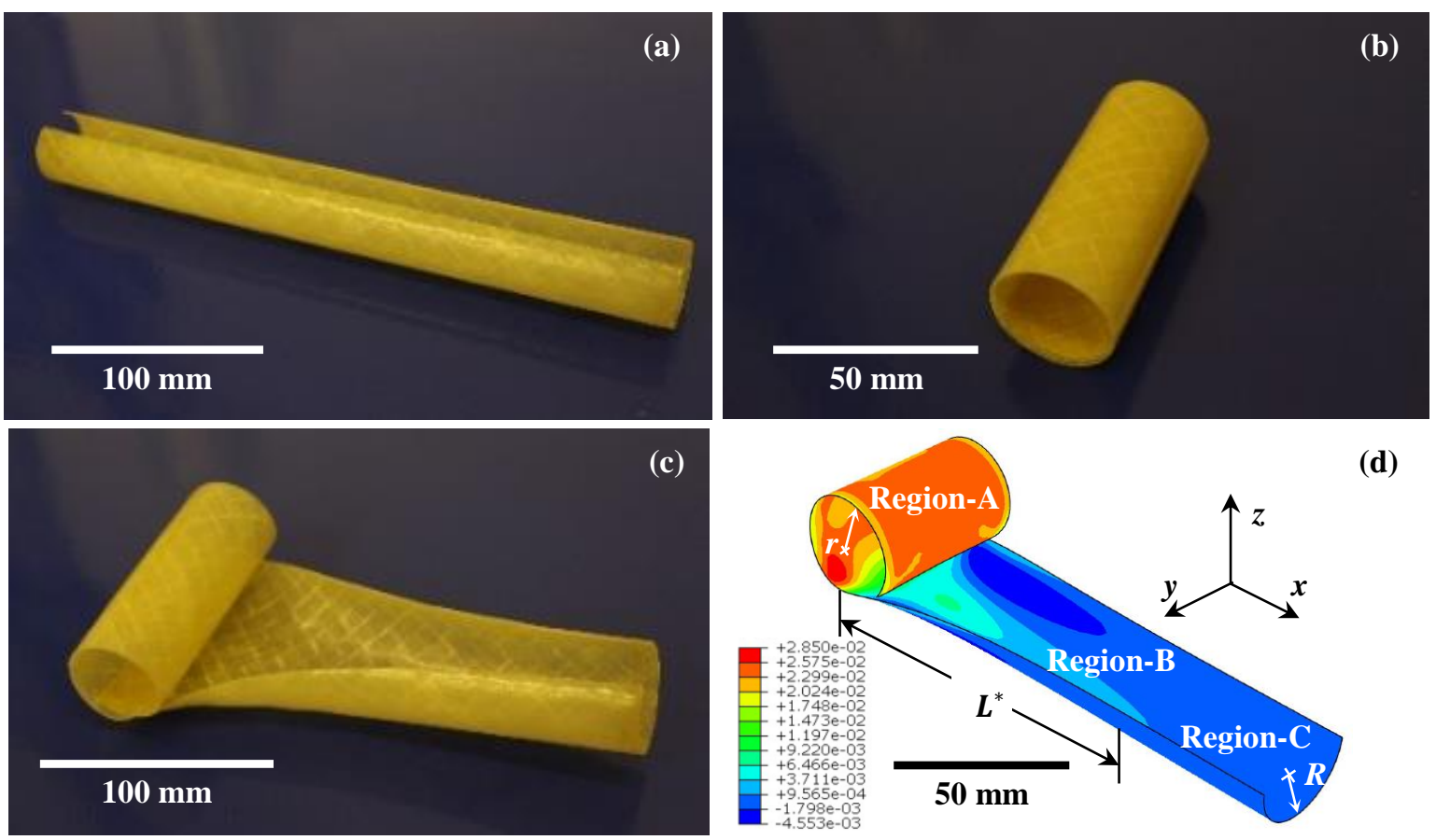

(d)

Figure 1 A bistable composite tape-spring based on GF/PP composite, showing (a) extended configuration; (b) coiled configuration; (c) intermediate ploy shape; (d) characterisation of ploy regions, contour is axial strain. 
We have characterised the inherent folding behaviour of the GF/PP-based CTS structures through experiments, finite element analysis and theoretical modelling [7]. A typical folding process consists of linear bending, torsional buckling, localisation and then folding at large displacements [8]. The shape of a folded CTS contains three regions, see Figures 1 (c) and (d): the central fold denoted as Region-A, which connects to the start of the ploy region, Region-B, over which the change in transverse curvature decays exponentially before returning to the undeformed straight end, Region-C [9]. The folded tape shape is dominated by axial strains and transverse curvature changes, and we have characterised the strain distribution along the folded tape in terms of experiments and FE simulation [10]: using a 3D strain mapping technique, data also show how the strains concentrate into features within the central fold Region-A, which are most likely to develop into "kinks". These kinks would induce undulation and inconsistency in folding, or even lead to premature failure of the CTS. Since fibres are oriented in $\pm 45^{\circ}$, axial tension or compression of the CTS corresponds to shear behaviour of the material. It is essential to study the shear failure mechanisms of the GF/PP composite to facilitate structural design of the CTS in aerospace applications.

Whilst there has been extensive research on microstructural analysis of a semi-crystalline polymeric material, the micromechanical evolution and progression of failure within a composite structure is rarely studied. Investigations into the latter would provide essential insight to understand the structure-property relationships of the composite, in order to ensure structural integrity of the CTS during the folding process, and ultimately assist the structural design and service life predictions of the CTS while in-service.

Non-destructive testing using X-rays or neutron diffraction offer special advantages over conventional destructive methods, and have been extensively used to investigate 
in-situ the microstructures, residual stresses, strain and stress distributions, deep inside a material system [11]. The strain measurements are based on monitoring the shifts of the Bragg's peak positions within a structure [12]. Although X-rays and neutron diffractions share similar principles, the neutron technique is superior in terms of penetration depth and light elements (with a larger neutron absorption cross-section) detection [13]. The ENGIN-X at Rutherford Appleton Laboratory, Science \& Technology Facilities Council (STFC), UK, is a dedicated time-of-flight (ToF) neutron diffractometer for strain measurements. The diffractometer offers a large detector coverage area and a wide scattering range, in order to provide data with high stability and accuracy [14]. Thus, it is an ideal tool to study the micromechanical shear failure of the GF/PP composite.

In this research, we investigate the micromechanical shear failure of the GF/PP composite at both macroscopic and microscopic scales. The macroscopic failure is characterised by a tensile failure of $\pm 45^{\circ}$ composite laminates; whilst microscopic failure is studied in-situ for different applied strain levels using ENGIN-X. The temperature dependency of the composite is also evaluated in the range from $-80{ }^{\circ} \mathrm{C}$ to $60{ }^{\circ} \mathrm{C}$. Here, we highlight the elastic and viscoelastic strain evolutions at both macroscopic and microscopic levels, in order to characterise the fundamentals of micromechanical shear failure of the CTS. Failure mechanisms are then proposed to provide further insight to benefit optimisation of structural design, as well as structural integrity and lifetime predictions of the CTS envisaged for aerospace applications.

\section{$2 \quad$ Theoretical}

\subsection{Neutron strain scanning}

Following the discovery of the neutron by Sir James Chadwick in Cambridge in 1932 [15], the neutron strain scanning (NSS) method was developed in the 1960s [11]. Figure 2 schematically shows an experimental setup for the neutron ToF scattering using 
ENGIN-X [14]. The neutron beam is pulsed over a wide range of kinetic energies and impinges a sample before being scattered by its material; detectors collect the diffracted neutrons at a fixed angle of $2 \theta_{\mathrm{b}}$. As neutrons can penetrate deep into a material, strains can be statistically and non-destructively measured. Assuming an elastic collision, the wavelength of the detected neutrons is defined by its ToF time, $t$ :

$$
\lambda=\frac{h}{m\left(L_{1}+L_{2}\right)} t
$$

where $h$ is the Planck's constant, $m$ is the neutron mass, and $L_{1}$ and $L_{2}$ are the primary and secondary flight paths, respectively, see Figure 2.

The spectrum diffracted by a polycrystalline material consists of different crystalline peaks corresponding to a Miller index $(h k l)$ family of lattice planes, and follow Bragg's law. Thus, the $d$-spacing is obtained from the position $t_{h k l}$ of the peak in the ToF spectrum, i.e.

$$
d_{h k l}=\frac{h}{2 \sin \theta_{\mathrm{B}} m\left(L_{1}+L_{2}\right)} t_{h k l}
$$

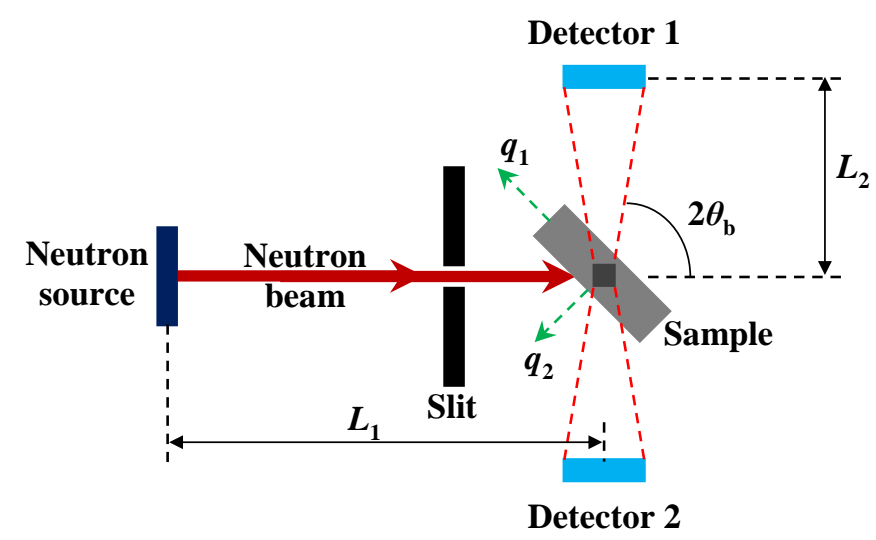

Figure 2 Schematic representation of the experimental setup at ENGIN-X; the elastic strain is measured along the directions of the impulse exchange vectors, $q_{1}$ (longitudinal direction) and $q_{2}$ (thickness direction), by the two detectors.

Peak positions are determined precisely by a least-squares refinement of the peaks, with a typical sensitivity of $50 \mu \varepsilon$ (microstrain). The elastic strain, $\varepsilon_{h k l}$, is calculated 
from the changes in the molecular interplanar distances $d_{h k l}$ along a general direction, compared with a stress-free reference, $d_{h k l}^{0}$, measured in the same direction [14]:

$$
\varepsilon_{h k l}=\left(d_{h k l}-d_{h k l}^{0}\right) / d_{h k l}^{0}
$$

\subsection{Phase identification}

To determine the microstructural phases of the GF/PP composite from neutron scattering, we first scanned pure GF and pure PP samples separately, which contain the same volume of material as in a GF/PP specimen. This showed that diffraction from GF has negligible effect on diffraction pattern of PP: the peak intensity $\left(n \AA^{-1} \mathrm{~s}^{-1} \mathrm{~mm}^{-3}\right)$ of pure GF pattern is only 9000 , compared to 230000 in a pure PP sample. Furthermore, the neutron pattern of pure PP is also found effectively to be the same as in a GF/PP sample.

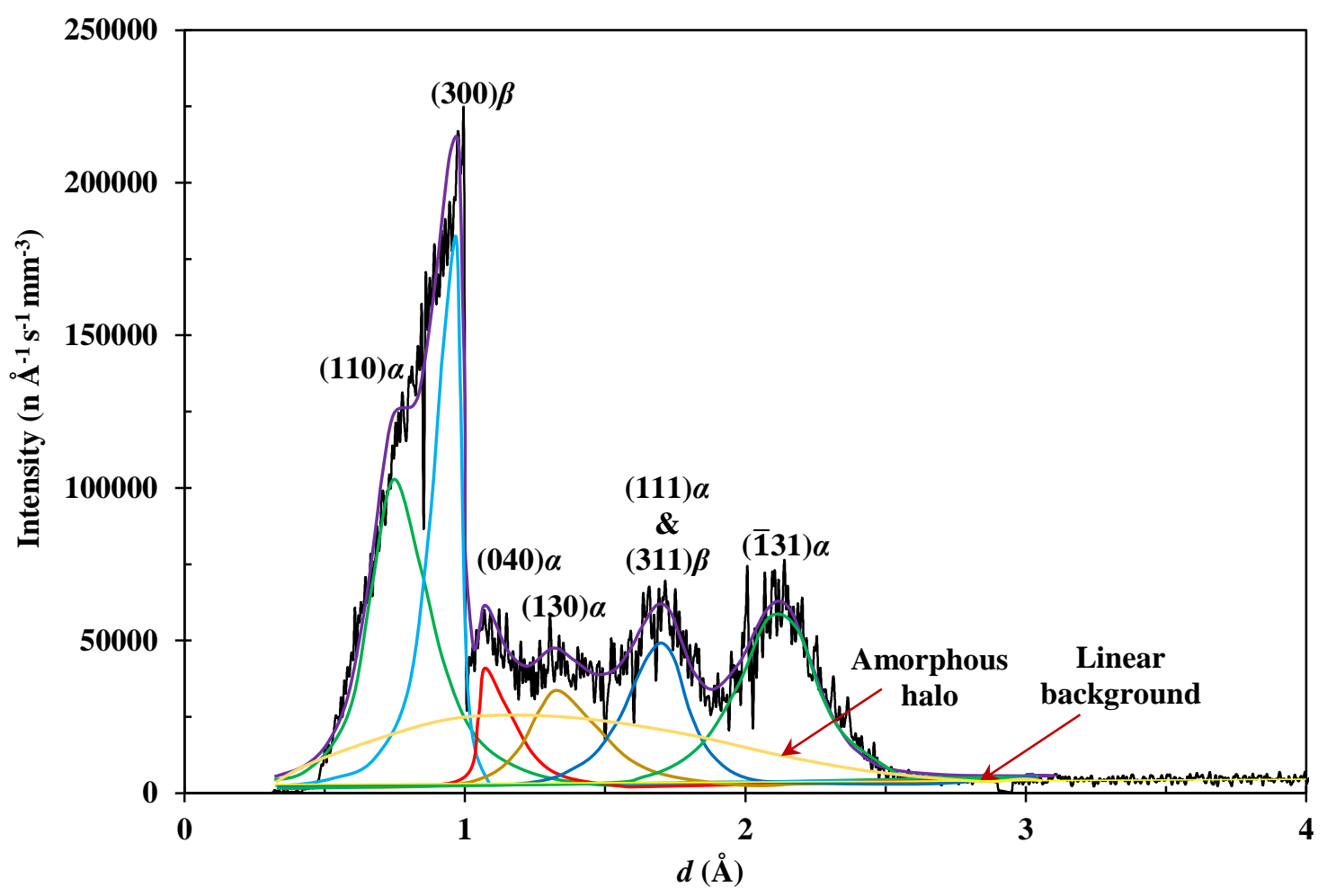

Figure 3 Profile analysis of neutron-scattering pattern of a stress-free composite sample using ENGIN-X.

The PP matrix is a semi-crystalline polymer with a melting temperature, $T_{\mathrm{m}}$, of $160{ }^{\circ} \mathrm{C}$ and a glass transition temperature $T_{\mathrm{g}}$ of $-10{ }^{\circ} \mathrm{C}$. Its microstructure has been extensively studied and well-understood [16-18]. The crystalline phase of PP varies from 
$\alpha$ - to $\gamma$-crystals, depending on the thermal conditions [19]. Generally, cooling of the PP at $50-300 \mathrm{~K} / \mathrm{s}$ leads to the formation of $\alpha$-crystals; at lower than $50 \mathrm{~K} / \mathrm{s}, \beta$-crystals grow. Faster cooling is associated with mesomorphic phase or vitrification of the entire melt [20]. The $\gamma$-crystals develop preferentially in the presence of chain defects, which limit the isotactic sequences, in low molar mass fractions or during crystallisation at an elevated pressure [21].

Figure 3 shows an example of profile analysis of the neutron scattering pattern of a GF/PP sample obtained using ENGIN-X. The raw data curve is smoothed first using MDI Jade 6.0 (Materials Data Inc., Livermore, CA, USA), and the background noise is maintained as linear. The function of the smoothed profile is based on Pearson's VII distribution, and is expressed as [22]:

$$
I_{2 \theta}=\frac{2 \Gamma(m)\left(2^{1 / m}-1\right)^{1 / 2}}{\sqrt{\pi} \Gamma(m-1 / 2)} \frac{I_{k}}{H_{k}}\left[1+4\left(\frac{2 \theta-2 \theta_{k}}{H_{k}}\right)^{2}\left(2^{1 / m}-1\right)\right]^{-m}
$$

Here, $m$ is the shape parameter, with a value of 1.52 , which has been proven to give the best fit for PP [23]. The integrated areas of the crystalline peaks can be used to calculate the degree of crystallinity:

$$
\text { Crystallinity }=\frac{\sum A_{\text {cri }}}{\sum A_{\text {cri }}+A_{\text {amo }}}
$$

where $\sum A_{\text {cri }}$ is the sum of integrated area of all the crystalline peaks, and $A_{\text {amo }}$ is the area of amorphous halo [19], which is a broad reflection peak from the amorphous region in Figure 3. Define the relative fraction of $\beta$-crystals, $k_{\beta}$, as:

$$
k_{\beta}=\frac{H_{\beta_{1}}}{H_{\beta_{1}}+H_{\alpha_{1}}+H_{\alpha_{2}}+H_{\alpha_{3}}}
$$

$H_{\alpha_{1}}, H_{\alpha_{2}}, H_{\alpha_{3}}$ are heights of the three strong equatorial $\alpha$-crystalline peaks with Miller indices corresponding to (110), (040) and (130), respectively; and $H_{\beta_{1}}$ is the height of the strong $\beta$-crystalline peak (300). Here, the representative $\beta$-peak is compared to the sum of the three $\alpha$-crystalline peaks rather than any one of these peaks, since the variation 
in the relative heights of three $\alpha$-peaks depends on the degree of isotaxy and the applied thermal treatment [19].

The microstructural phases within a GF/PP composite can be determined as indicated in Figure 3. Upon cooling, the PP matrix crystallises into $\alpha$-crystals. Since the surfaces of oriented $\alpha$-crystals can trigger the formation of $\beta$-crystals [24], the oven-baking process results in a mixture of $\alpha$ - and $\beta$-crystals; the peak positions are also identified [19]. The peak reflections from $\alpha$-crystals include planes with indices of (110), (040), (130), (111) and (131); $\beta$-reflection peaks include (300), and (311). The latter coincides with the $\alpha$-(111) reflection and is invisible; the (300) is a distinctive and strong reflection that usually represents $\beta$-crystals [25]. The crystallinity and fraction of $\beta$-crystals within a stress-free GF/PP composite can be determined using Eqns 5 and 6, and they are found to be $70.9 \%$ and $51.0 \%$, respectively.

\subsection{Elastic and viscoelastic strain}

For an orthotropic composite laminate under plane stress state, its stress-strain is expressed as:

$$
\left[\begin{array}{c}
\varepsilon_{11}(t) \\
\varepsilon_{22}(t) \\
\gamma_{12}(t)
\end{array}\right]=\left[\begin{array}{ccc}
S_{11} & S_{12} & 0 \\
S_{21} & S_{22} & 0 \\
0 & 0 & S_{66}(t)
\end{array}\right]\left[\begin{array}{c}
\sigma_{1}(t) \\
\sigma_{2}(t) \\
\tau_{12}(t)
\end{array}\right]+\left[\begin{array}{c}
0 \\
0 \\
\gamma_{12}(t)
\end{array}\right]_{v i s}
$$

where $S_{\mathrm{ij}}$ are the elements of the compliance matrix; the subscript vis is the viscoelastic term of strain. The in-plane shear properties of a composite can be determined through a uniaxial tensile test of $\pm 45^{\circ}$ laminates following ASTM D3518 [27], thus:

$$
\gamma_{12}(t)=\tau_{12}(t) / G_{12}+\gamma_{12}(t)_{v i s}
$$

The compliance in the fibre direction is virtually independent of time effects; the compliance components $S_{12}$ and $S_{21}$ have weak time dependencies [26]. Thereby, the fibre-dominated compliance terms $\left(S_{11}=S_{22}, S_{12}=S_{21}\right)$ are assumed to be time independent, whilst the matrix dominated terms $\left(S_{66}\right)$ are time dependent. 


\subsection{Composite sample preparation}

Production of composite samples followed the process previously described [8]. Briefly, the layup consists of three layers of plain-weave GF and PP sheet, and Table 1 gives their properties and the composite sample dimensions. The layup was then placed between two pieces of PTFE coated glass fabric, and gradually wrapped and tightened on a flat mould using heat-shrink tape. Foldback clips were used to lock the layup before curing in a pre-heated fan-assisted oven at $205{ }^{\circ} \mathrm{C}$ for 4 hours. After releasing the mould, each sample was manually cut through waterjet into designated size (inset in Table 1) in readiness for testing; the composite has a fibre volume fraction of $30 \%$.

Table 1 Material properties of GF fabrics and PP sheets, as well as the composite sample dimensions for testing.

\begin{tabular}{|c|c|c|c|c|}
\hline Materials & GF fabric & PP sheet & & 28,92 \\
\hline Density & $200 \mathrm{~g} / \mathrm{m}^{2}$ & $0.9 \mathrm{~g} / \mathrm{cm}^{3}$ & & \\
\hline Thickness (mm) & 0.20 & 0.5 & & \\
\hline Construction warp $\times$ weft $(\mathrm{th} / \mathrm{cm})$ & $7.4 \times 7.4$ & -- & t & 20 \\
\hline Type of yarn & EC9 $68 \times 2$ & -- & & \\
\hline Poisson's ratio & 0.2 & 0.42 & ¿ & \\
\hline Elastic modulus (GPa) & 72.4 & 1.53 & & \\
\hline Shear modulus (GPa) & 30 & 0.54 & I & \\
\hline $\begin{array}{l}\text { Thermal expansion coefficient } \\
\left(\mu \mathrm{m} / \mathrm{m}^{\circ} \mathrm{C}\right)\end{array}$ & 5.0 & 84.8 & 0 & \\
\hline
\end{tabular}

\subsection{Macroscopic failure testing}

Macroscopic shear failure of the GFPP composite was performed at the ENGIN-X following ASTM D3518 [27]. Figure 4 shows the experimental setup. Composite samples $\left( \pm 45^{\circ}\right.$ fibre layup) were attached to the tensile grips within an environmental chamber through bespoke aluminium adapters, which provided a clear span of $30 \mathrm{~mm}$. An extensometer and a $k$-type thermocouple were mounted on the sample surface for data monitoring. All tests were performed at a crosshead speed of $2 \mathrm{~mm} / \mathrm{min}$, with 
temperatures ranging from -80 to $60{ }^{\circ} \mathrm{C}$. Owing to the limitations of space inside the environmental chamber, the applied strain level was limited to $30 \%$.

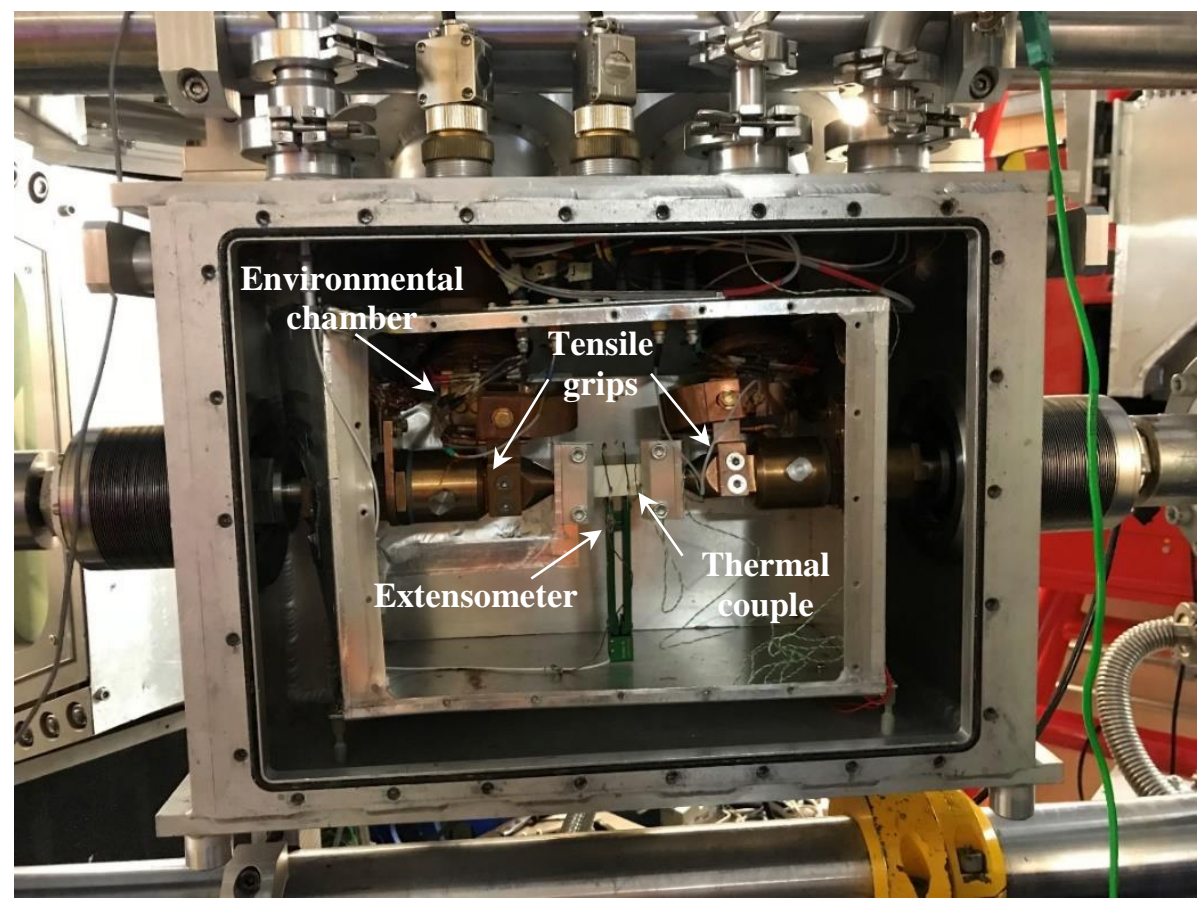

Figure 4 Experimental setup for shear failure tests inside an environmental chamber at EINGIN-X, STFC.

\subsection{Microscopic failure testing}

The neutron strain scanning (NSS) was performed using the ENGIN-X diffractometer. The strains and temperatures were applied similarly to those in Section 3.2 where now the neutron beam was applied at designated conditions, for certain strain and temperature combinations, see Figure 5. The neutron beam was a polychromatic (white) beam with a range of neutron wavelengths, $0.5 \AA-6 \AA$ for its ToF nature. The neutron beam was incident at $45^{\circ}$ to the longitudinal axis of the sample, corresponding to a scattering angle of $2 \theta=90^{\circ}$, see Figure 2, the gauge volume of the applied neutron beam was $4 \times 4 \times 10 \mathrm{~mm}$; again, the temperature range was $-80{ }^{\circ} \mathrm{C}$ to $60{ }^{\circ} \mathrm{C}$. For all tests, the stress-free datum lattice parameters $\left(d_{\mathrm{hkl}}, 0\right)$ were measured without constraints on samples for pure PP (before and after heat treatment), pure GF and the GF/PP composite. 


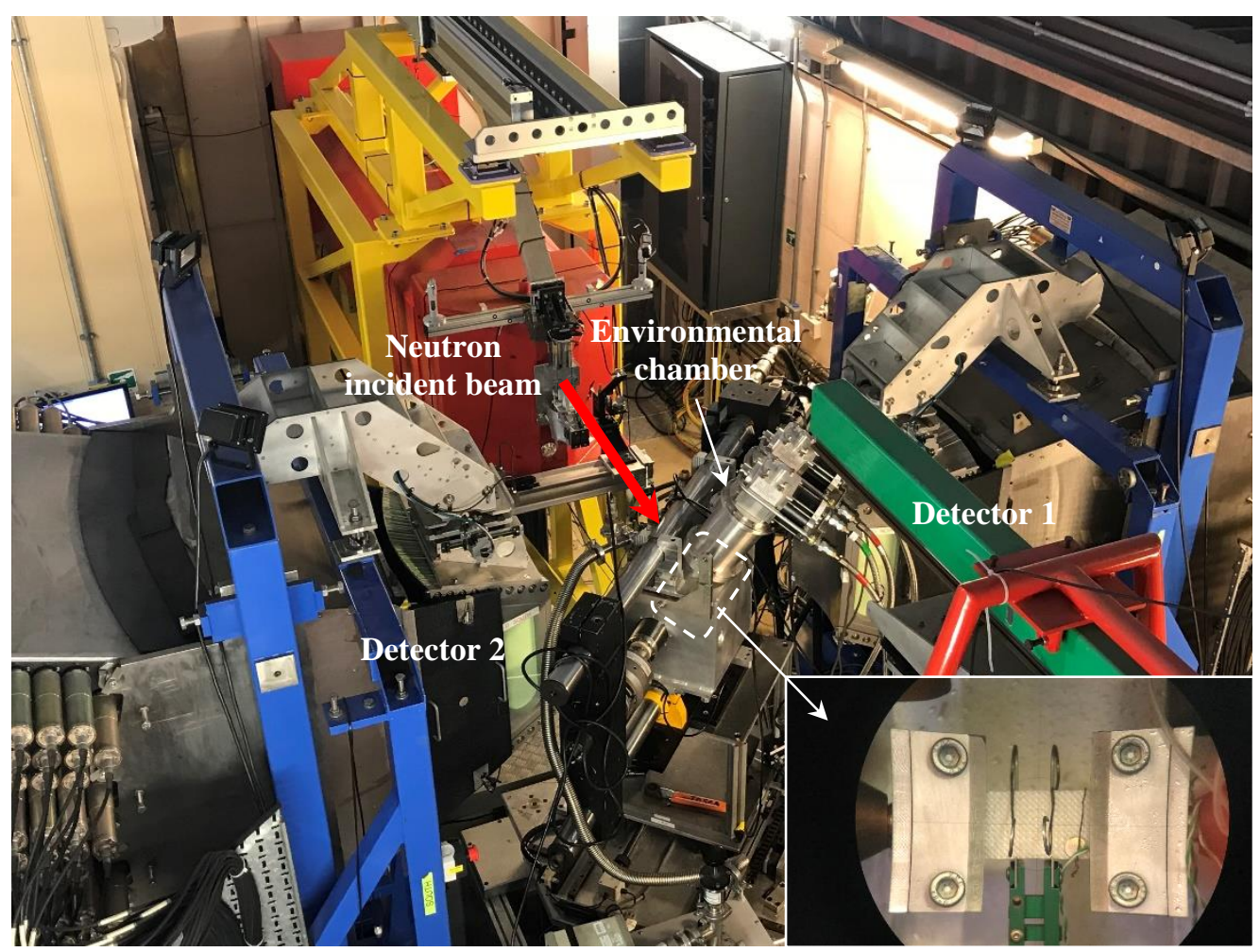

Figure 5 Experimental setup for the neutron scattering during shear failure tests of the GF/PP samples at EINGIN-X. Inset image shows the bespoke tensile rig with a sample inside the environmental chamber.

\section{Results and discussion}

\subsection{Residual strain}

Since the reinforcement from GF has marginal effect on the neutron pattern, the residual stain within the composite can be evaluated through microstructural changes within the PP matrix. The oven-baking process corresponds to a recrystallisation of PP, where the sample was found to have a crystallinity of $73.3 \%$, and $\beta$-crystal ratio of $53.4 \%$. For the cooled composite, there is slight reduction in both crystallinity and $\beta$-crystal ratio of $70.9 \%$ and $51.0 \%$, respectively. A comparison of neutron patterns from both samples shows slight peak shifts of the (111) and (131) crystalline planes, which are due to $\alpha$-crystals, indicating that residual stresses introduce tension in the composite. The residual strain associated with these two peaks are $3 \%$ and 5\%, respectively, and the corresponding residual stresses are caused by mismatch of thermal expansion coefficients between fibre and matrix materials during cooling [28]. 


\subsection{Macroscopic shear failure}

Figure 6 shows the stress-strain curve in shear of a GF/PP sample at room temperature. The shear modulus, $G_{12}$, is calculated from the gradient of the quasi-elastic linear region to be $851 \mathrm{MPa}$, which is about $3 \%$ of experimental error with our previous shear failure characterisation using embedded strain gauges [8,29]. The profile then becomes nonlinear, followed by a second, almost linear response, with a tangent shear modulus, $G_{12}^{*}=87.6 \mathrm{MPa}$, before fracture and failure occurs: the corresponding shear strength and shear strain are $48.0 \mathrm{MPa}$ and $27.3 \%$, respectively.

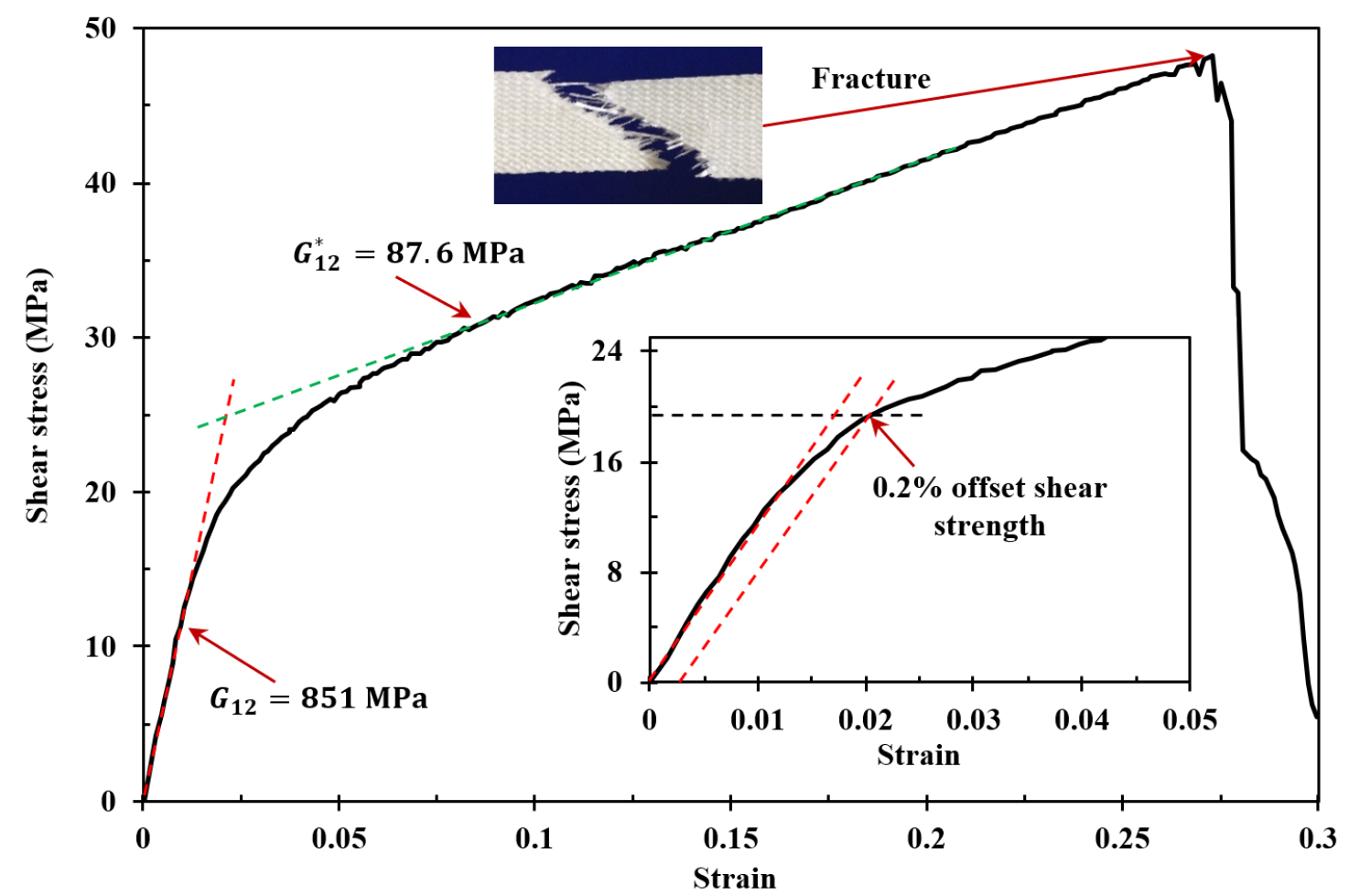

Figure 6 Stress-strain curve of a GF/PP sample in shear at room temperature; inset shows the definition of $0.2 \%$ offset shear strength, following ASTM D3518 [27].

The yield shear stress and strain can be determined by shifting the initial quasi-elastic line by $0.2 \%$ along the strain axis, as recommended by ASTM D3518 [27]. Thus, the transition from elastic to onset of the viscoelastic deformation is at an absolute strain of 2.2\%, with a yield stress of 19.0 MPa, see Figure 6 . 


\subsection{Macroscopic temperature dependency}

Figures 7 (a) and (b) show the temperature dependency of the shear modulus, $G_{12}$, tangent shear modulus, $G_{12}^{*}, 0.2 \%$ offset shear strength, and shear strain at failure determined from the profile scheme in Figure 6. Linear regressions can be fitted with a minimum correlation coefficient of 0.96 . Both the shear modulus and the shear yield strength decrease with increasing temperature; the shear strain at failure increases with temperature, which is attributed to the softening of molecular chains at increased temperatures - this is further discussed in Section 4.4, for the microscopic scale.

Figure 7 (c) shows the temperature dependency of viscoelastic strain calculated via Eqn 8. It shows that the initial linear regions are purely elastic for all tested temperatures, giving close-to-zero viscoelastic strain. Shear behaviour of the composite shows time dependent which is dominated by the matrix (through viscoelasticity) as discussed in Section 2.3. The secondary linear region correlates to a significant increase in viscoelastic strain, which is consistent with the linear viscoelasticity of the GF/PP composite characterised by experimental stress relaxation tests [30]. In the transition region between $G_{12}$ and $G_{12}^{*}$, the trend cannot be clearly identified within the temperature range of $-80{ }^{\circ} \mathrm{C}$ to $60{ }^{\circ} \mathrm{C}$ since they cross-over at different points, whilst they all fall within the strain range of 2 to $3 \%$.

\subsection{Microstructural shear failure}

Figure 8 (a) shows an example of the comparison between different neutron patterns of GF/PP composite at various strain levels at room temperature. Generally there are clear peak shifts of (111) and (131) crystalline planes from $\alpha$-crystals during loading. The diffraction peak (300) from $\beta$-crystals are stable across all of the applied strain levels since $\beta$-crystals are primarily un-oriented [25]. Thus, the elastic strain in the micromechanical evolution analysis is mainly focused on $\alpha$-crystals. 
The crystalline peak positions are from the tested samples in the longitudinal direction ( $q_{1}$ in Figure 2$)$. The elastic strain carried by $\alpha$-crystals can be calculated using Eqn 3, and the resulting data are shown in Figures 8 (b) to (d). The premature failure of crystals was determined from the neutron pattern where the peak splits into two was clearly observed at a certain strain value.
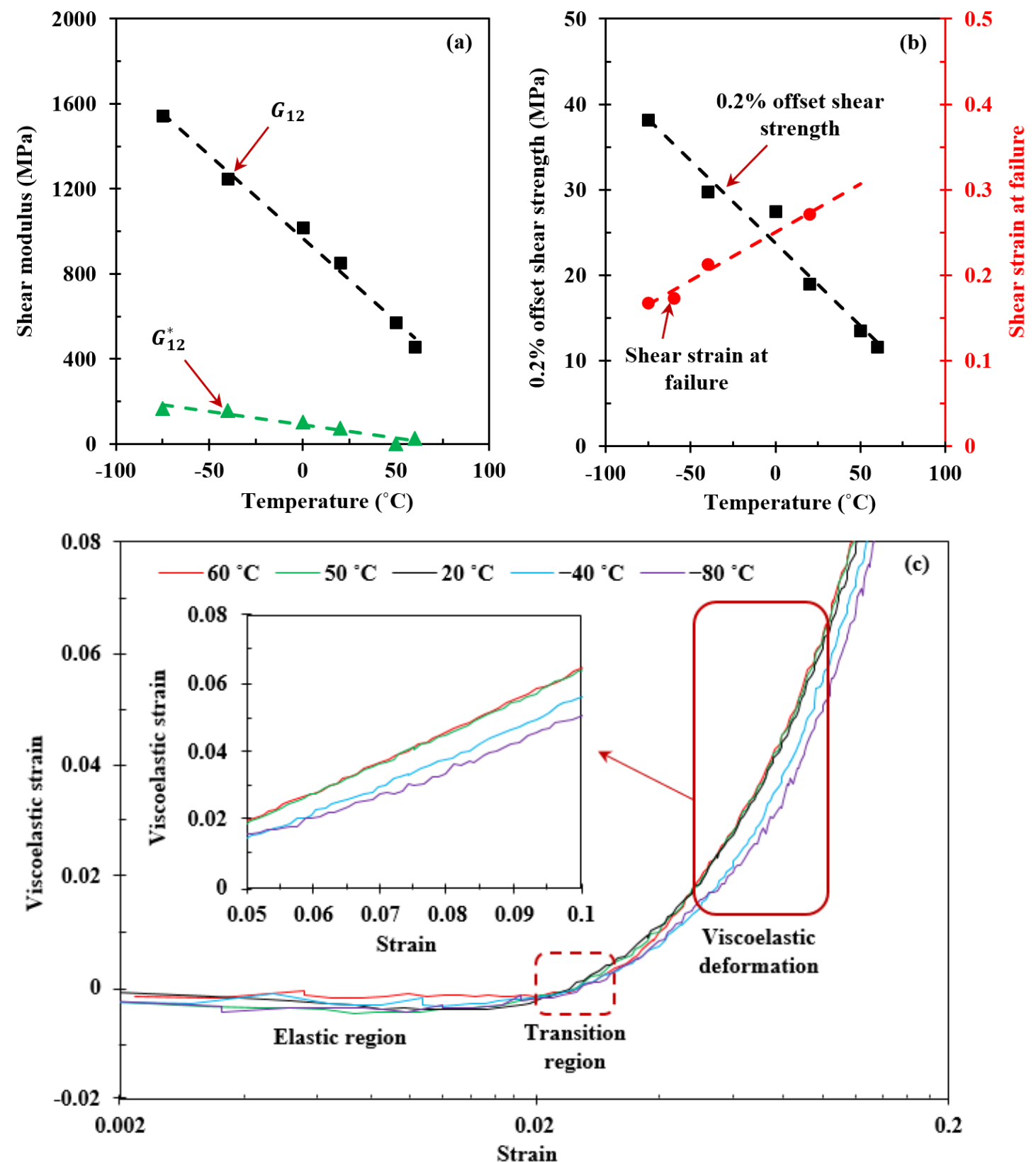

Figure 7 Macroscopic temperature dependency of (a) shear moduli $\left(G_{12}\right.$ and $\left.G_{12}^{*}\right)$; (b) $0.2 \%$ offset shear strength and shear strain at failure; and (c) viscoelastic strain. 
As shown in Figure 8 (b), the initial strain carried by the crystalline regions increases almost linearly with the applied macroscopic strain. Fracture was then observed at an applied strain of $15 \%$, corresponding to a significant drop in crystallinity in Figure 8 (c); the strain in the crystalline regions becomes saturated and stabilised at around 14\%. Upon initial loading, it is also observed that there is an increase in the $\beta$-crystal ratio in Figure 8 (d); applying shear promotes the growth of $\beta$-crystals [31], which then decreases with the applied strain level.

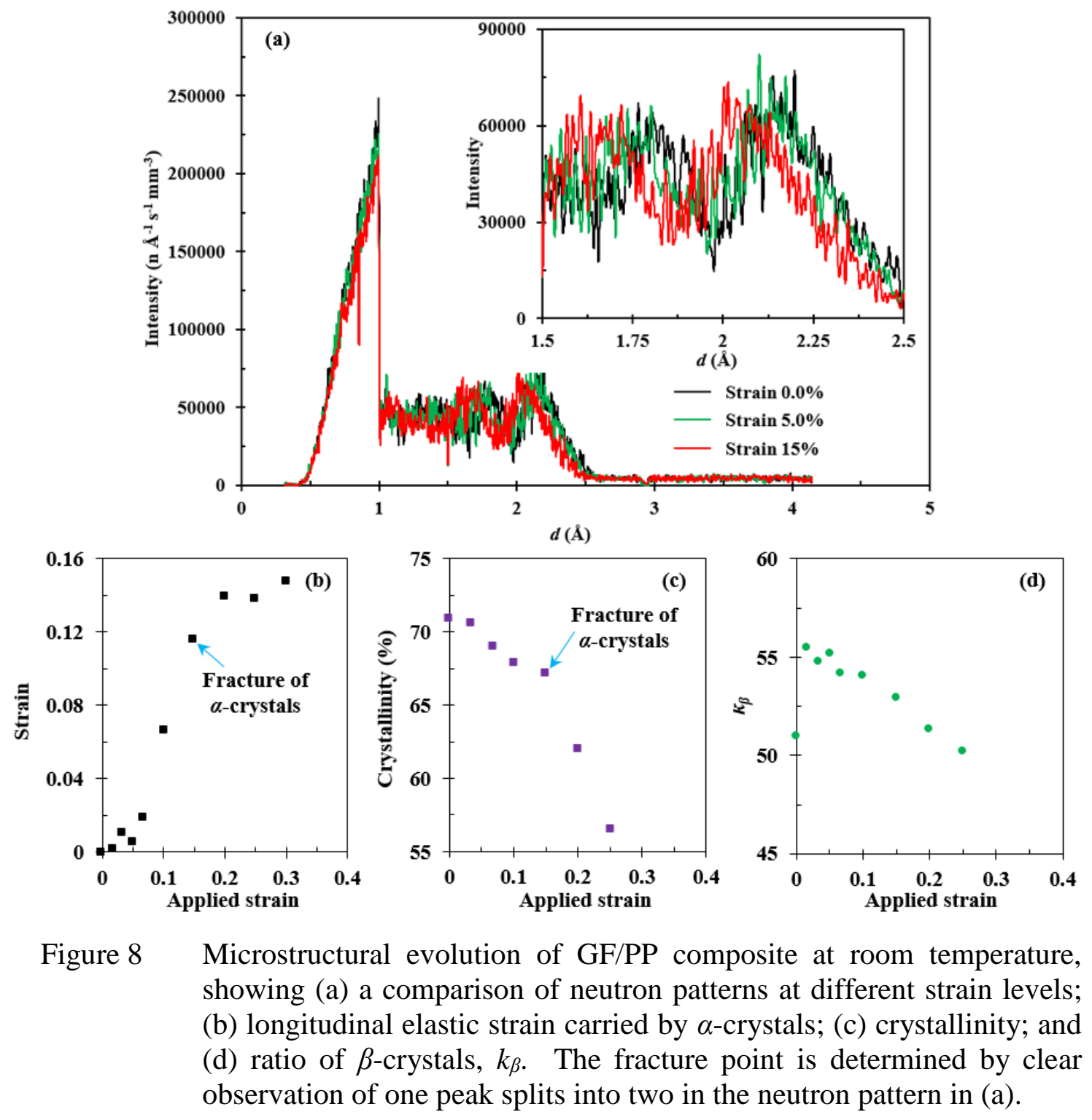


The elastic and viscoelastic strain evolutions at different temperatures are shown in Figure 9. There are clear differences in crystalline behaviour above and below the glass transition temperature of $\mathrm{PP}\left(-10^{\circ} \mathrm{C}\right)$ indicating that the micromechanical shear failure mechanisms of the composite are also temperature dependent.
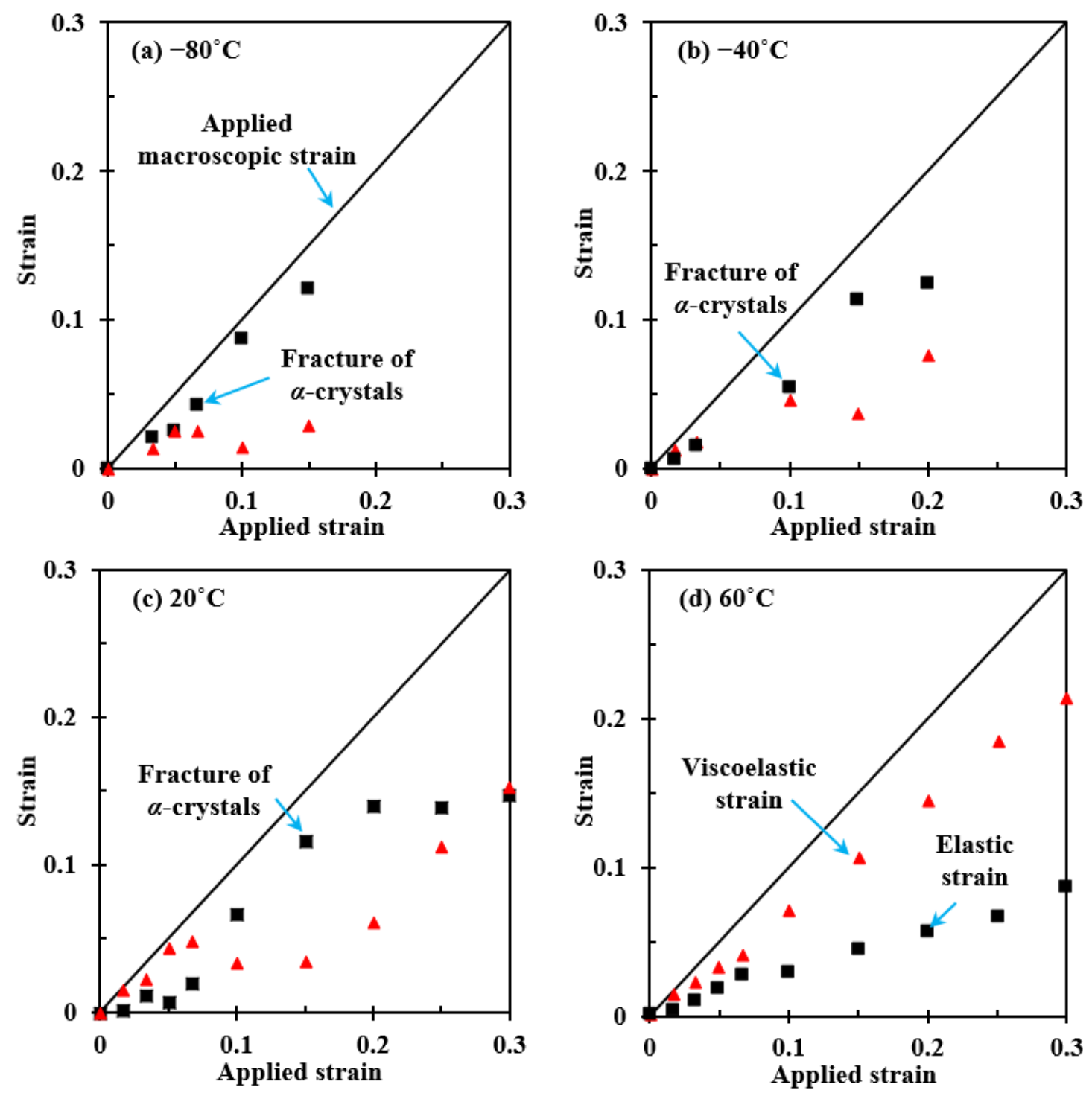

Figure 9 Elastic and viscoelastic strain evolutions in shear failure of GF/PP composite at temperature of (a) $-80{ }^{\circ} \mathrm{C}$; (b) $-40{ }^{\circ} \mathrm{C}$; (c) $20^{\circ} \mathrm{C}$; (d) $60{ }^{\circ} \mathrm{C}$. Squares: elastic strain; triangles: viscoelastic strain component.

For a semi-crystalline material, a two-phase microfibrillar model was developed by Peterlin [32], and is generally considered to be applicable. Here, crystals and amorphous regions are bridged by taut-tie molecular chains (TTMs), which have a distribution of 
contour lengths or strains at breaking, and carry loads when stressed [33]. Later, the TTMs were considered to form the oriented amorphous phase, as in the three-phase or so-called "Swiss-cheese" model [34], and the mechanical performance (i.e. stiffness and toughness) is proved to be controlled by these TTMs $[35,36]$.

Below the glass transition temperature, in Figures 9 (a) and (b), most of the molecules cannot move, and the composite material is stiffer, resulting in higher modulus values, c.f. Figure 7. Crystals are also more effective in carrying loads, giving a proportional response to applied macroscopic strains, until failure. Since the crystalline regions occupy approximately $70 \%$ in volume, they carry most of the applied strain, which also reduces with increased temperature. When crystals start to fracture, the GF/PP composite still behaves as an elastic solid with viscoelastic strains remaining small.

Above the glass transition temperature and at low macroscopic applied strain i.e. from 0 to $5 \%$, the crystalline regions are resistant to mechanical loading, with only $0.6 \%$ of strain carried by the $\alpha$-crystals when the applied strain is $5 \%$, at room temperature (Figure 9-c). This indicates that initial loads are carried by TTMs, as they determine instant load-carrying ability [33]; increasing the applied strain levels from 5\% to $15 \%$, load transitions occur within all three phases. Elastic deformations are carried by crystalline regions as indicated by the linear strain change, Figure 9-c, and residual strains are stored in the amorphous phases, which control the viscoelasticity [36]. Further increases in macroscopic strain lead to fracture of crystals, signifying the maximum capacity of $\alpha$-crystals; there is then a significant drop in crystallinity, and additional deformation relies mainly on amorphous regions as the crystalline strain becomes saturated and stabilised. At $60{ }^{\circ} \mathrm{C}$ in Figure 9 (d), viscoelastic strain increases faster than elastic strain, and they both show close-to-linear growth with applied strain. This infers that the TTMs are softening, and all the regions respond collectively to the straining. 


\subsection{Multiscale shear failure mechanisms}

Despite the microscopic premature failure of crystals, the observed maximum strain carried in crystals non-linearly increases with temperature, see Figure 10 (a): molecules in both the amorphous and crystalline regions become soft at higher temperatures, and contribute to the elongation capability of the composite. On the other hand, data scattering at lower temperatures is observed which may be caused by the unstable crack propagation below the glass transition temperature since the molecules are brittle [37].
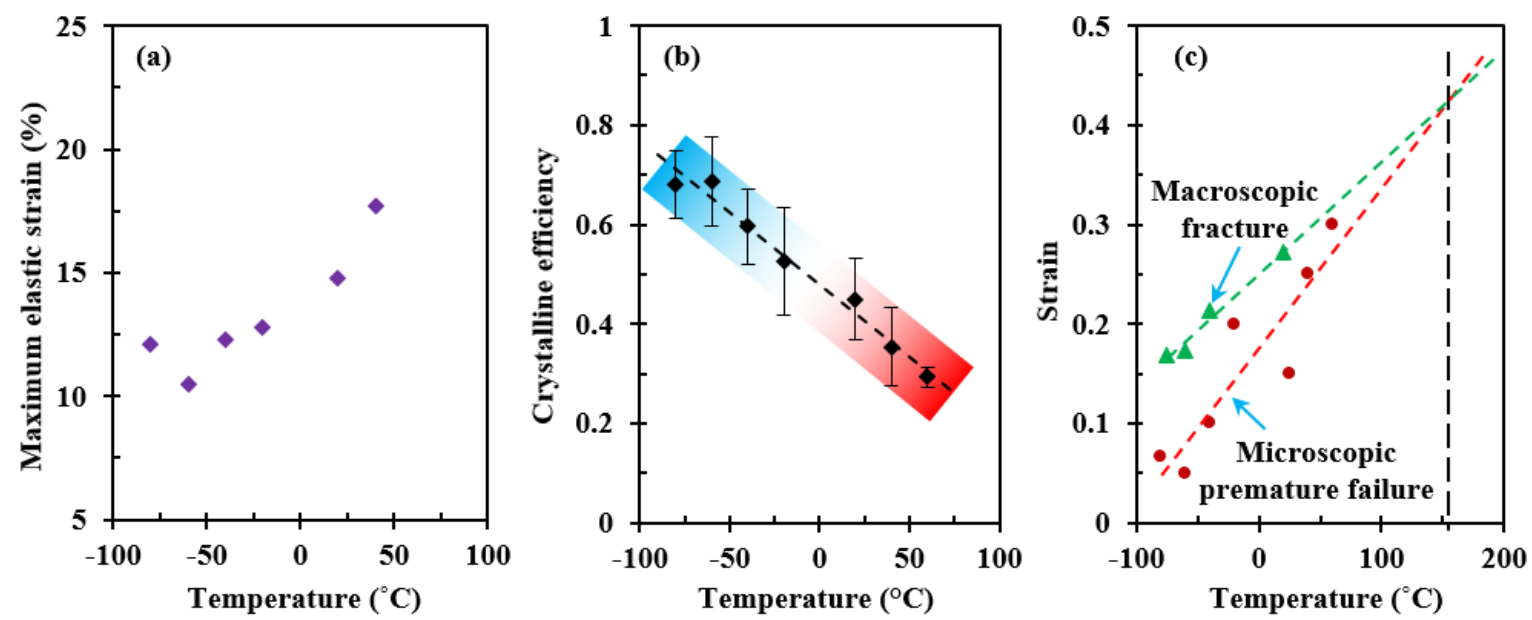

Figure 10 (a) Maximum elastic strain carried by $\alpha$-crystals at different temperature; (b) crystalline efficiency at various temperatures, error bars are standard errors; (c) shear failure strain evolution at both macro- and micro-scales.

Defining the crystalline efficiency as the proportion of mean percentage of elastic strain carried by the crystalline regions to the applied macroscopic strain for each case, we plot its statistical relationship with temperature in Figure 10 (b). Since most of the polymeric matrices are temperature dependent, as evidenced in Section 4.5, we assume that the crystalline efficiency is also temperature dependent, i.e. it is constant at each temperature, and the discrepancies may be attributed to the minor effects from potential phase transitions at different strain levels. A linear regression is applied with a correlation coefficient of 0.99 , to reveal parameter values:

$$
\text { Crystalline efficiency }=-2.9 \times 10^{-3} \times \text { Temperature }\left({ }^{\circ} \mathrm{C}\right)+0.48
$$


The linear regression of Eqn 9 is extrapolated forward and backward, then we observe the following terms when the efficiency is zero and unity, respectively.

An efficiency of unity correlates to the strains carried entirely by crystalline region, with the composite behaving as a pure elastic solid at a temperature of $-180{ }^{\circ} \mathrm{C}$; whilst a zero value correlates to zero load-bearing ability at a temperature of $165^{\circ} \mathrm{C}$. Considering that the melting temperature of $\mathrm{PP}$ is $160{ }^{\circ} \mathrm{C}$ [38], the linear regression is effective in predicting the load bearing efficiency of the crystalline regions, and the parameter values in Eqn 9 are material dependent. The linear temperature dependency may also be closely related to the linear viscoelasticity of the GF/PP composite [30].

Figure 10 (c) compares the changes in failure strain from both the composite samples and the $\alpha$-crystals i.e. failure at the macroscale versus premature microscale failure. Again, a linear regression is fitted for both curves, resulting in an intersection point at $152{ }^{\circ} \mathrm{C}$ : this is close to the melting temperature of the $\mathrm{PP}\left(160{ }^{\circ} \mathrm{C}\right)$, which confirms the effectiveness of the linear fitted macroscopic and microscopic failure strains.

\section{$5 \quad$ Conclusions}

We have investigated in-situ the micromechanical shear failure of a GF/PP composite using neutron diffraction at the ENGIN-X of Rutherford Appleton Laboratory, STFC, UK. The shear failures at both macroscopic and microscopic levels have been determined to provide further insight into the failure mechanisms of the GF/PP based CTS structure.

The residual strain within the composite is first characterised. The micromechanical shear failure of the GF/PP composite is dominated by the PP matrix, which follows the three-phase microstructural model of crystalline, amorphous and oriented amorphous phases. There are clear observations of microstructural phase changes upon straining. The elastic and viscoelastic strain evolutions reveal the 
fundamentals of micromechanical shear failure: it is temperature-dependent, showing clear differences above and below the glass transition temperature. The premature failure of crystalline regions has also been determined through in-situ neutron diffraction, and correlates well with the macroscopic shear failure. Since macroscopic yielding occurs before the appearance of any microstructural fractures, we conclude that yielding is controlled by the TTMs. The load-bearing efficiency of the crystalline regions has been determined, which varies with temperature: crystalline regions carry $100 \%$ of loads at $-180{ }^{\circ} \mathrm{C}$ since the molecules are "frozen', and have zero load capability at $165^{\circ} \mathrm{C}$ when approaching the melting temperature of the matrix.

Since GF/PP composite material is the most-established material system of the bistable CTS structure, these findings provide valuable information and essential data to benefit the structural design, optimisation, and maintenance of structural integrity of the CTS during folding. Future work will focus on temperature-dependent viscoelastic behaviour, endurance analysis, as well as sizing and load-carrying capacities of the CTS, in order to facilitate its applications and service life predictions for aerospace engineering.

\section{Acknowledgements}

The authors would like to thank the financial support from Innovate UK for "Large Landing Gear of the Future: Bistable Composite Technologies'" (Grant No. 113077, RG82506), in close partnership with SAFRAN Landing Systems Ltd., UK. The award of the ENGIN-X beamtime (RB1910213) from the ISIS Neutron and Muon Source, Rutherford Appleton Laboratory, Science and Technology Facilities Council (STFC), UK, is also acknowledged. This work is also supported by the technical staff and aegis of the National Research Facility for Infrastructure Sensing (NRFIS, Grant No. EP/P013848/1), Cambridge University Engineering Department.

\section{References}

[1] Daton-Lovett A. An extendible member. PCT/GB97/00839, 1996. 
[2] Iqbal K, Pellegrino S. Bi-stable composite shells. Proceeding 41st AIAA/ASME/ASCE/ AHS/ASC Struct. Struct. Dyn. Mater. Conf. Exhib., Atlanta: 2000, p. 1-8.

[3] Galletly DA, Guest SD. Bistable composite slit tubes. I. A beam model. Int J Solids Struct 2004; 41: 4517-33.

[4] Yee JCH, Pellegrino S. Folding of woven composite structures. Compos Part A Appl Sci Manuf 2005; 36: 273-8.

[5] Guest SD, Pellegrino S. Analytical models for bistable cylindrical shells. Proc R Soc A Math Phys Eng Sci 2006; 462: 839-54.

[6] Schmidt RK. Aircraft landing gear assembly. EP3069991B1, 2017.

[7] Seffen KA, Wang B, Guest SD. Folded orthotropic tape-springs. J Mech Phys Solids 2019; 123: $138-48$.

[8] Wang B, Seffen KA, Guest SD. Folding of bistable composite tape-springs. University of Cambridge, Department of Engineering; 2019. doi:10.17863/CAM.36403.

[9] Wang B, Seffen KA, Guest SD. Shape of a bistable composite tape-spring in folding. AIAA SciTech 2019 Forum, San Diego: 2019, p. 1-12.

[10] Wang B, Seffen KA, Guest SD. Folded strains of a bistable composite tape-spring. 2020 (drafted).

[11] Fitzpatrick ME, Lodini A. Analysis of residual stress by diffraction using neutron and synchrotron radiation. CRC Press; 2003.

[12] Hutchings MT, Withers PJ, Holden TM, Lorentzen T. Introduction to the characterization of residual stress by neutron diffraction. CRC press; 2005.

[13] Wang B, Zhong S, Lee T, Fancey KS, Mi J. Non-destructive testing and evaluation of composite materials/structures: A state-of-the-art review. Adv Mech Eng 2020; 12(4): 1-28.

[14] Santisteban JR, Daymond MR, James JA, Edwards L. ENGIN-X: a third-generation neutron strain scanner. J Appl Crystallogr 2006; 39: 812-25.

[15] Chadwick J. Possible existence of a neutron. Nature 1932; 129: 312.

[16] Natta G, Corradini P. Structure and properties of isotactic polypropylene. Nuovo Cim 1960; 15: 40-51.

[17] Stamm M, Schelten J, Ballard D. Determination of the chain conformation of polypropylene in the crystalline state by neutron scattering. Colloid Polym Sci 1981; 259: 286-92.

[18] Meille SV, Brückner S. Non-parallel chains in crystalline $\gamma$-isotactic polypropylene. Nature 1989; 340: 455.

[19] Turner-Jones A, Aizlewood JM, Beckett DR. Crystalline forms of isotactic polypropylene. Die Makromol Chemie Macromol Chem Phys 1964; 75: 134-58.

[20] Mollova A, Androsch R, Mileva D, Gahleitner M, Funari SS. Crystallization of isotactic polypropylene containing beta-phase nucleating agent at rapid cooling. Eur Polym J 2013; 49: $1057-65$.

[21] Turner-Jones A. Development of the $\gamma$-crystal form in random copolymers of propylene and 
their analysis by dsc and x-ray methods. Polymer 1971; 12: 487-508.

[22] Hall MM, Veeraraghavan VG, Rubin H, Winchell PG. The approximation of symmetric Xray peaks by Pearson type VII distributions. J Appl Crystallogr 1977; 10: 66-8.

[23] Immirzi A. Constrained powder-profile refinement based on generalized coordinates. Application to X-ray data of isotactic polypropylene. Acta Crystallogr Sect B 1980; 36: 237885 .

[24] Varga J, Karger-Kocsis J. Interfacial morphologies in carbon fibre-reinforced polypropylene microcomposites. Polymer 1995; 36: 4877-81.

[25] Somani RH, Hsiao BS, Nogales A, Fruitwala H, Srinivas S, Tsou AH. Structure development during shear flow induced crystallization of i-PP: In situ wide-angle X-ray diffraction study. Macromolecules 2001; 34: 5902-9.

[26] Sullivan JL. Creep and physical aging of composites. Compos Sci Technol 1990; 39: 207-32.

[27] ASTM International. ASTM D3518 - Standard test method for in-plane shear response of polymer matrix composite materials by tensile test of a $\pm 45^{\circ}$ laminate 2013 : 1-7.

[28] Hull D, Clyne TW. An introduction to composite materials. Cambridge university press; 1996.

[29] Wang B, Seffen KA, Guest SD. Folding of a bistable tape-spring structure based on plainwoven composite. 26th Int Conf Compos Eng 2018: 1-2.

[30] Wang B, Seffen KA, Guest SD, Lee T, et al. In-situ studies of failure mechanisms of a bistable composite tape-spring under extreme conditions. ISIS Neutron Muon Source Data J 2019.

[31] Huang MR, Li XG, Fang BR. $\beta$-nucleators and $\beta$-crystalline form of isotactic polypropylene. J Appl Polym Sci 1995; 56: 1323-37.

[32] Peterlin A. Molecular model of drawing polyethylene and polypropylene. J Mater Sci 1971; 6: 490-508.

[33] Peterlin A. Structural model of mechanical properties and failure of crystalline polymer solids with fibrous structure. Int J Fract 1975; 11: 761-80.

[34] Prevorsek DC, Harget PJ, Sharma RK, Reimschuessel AC. Nylon 6 fibers: Changes in structure between moderate and high draw ratios. J Macromol Sci Part B 1973; 8: 127-56.

[35] Marcellan A, Bunsell AR, Laiarinandrasana L, Piques R. A multi-scale analysis of the microstructure and the tensile mechanical behaviour of polyamide 66 fibre. Polymer 2006; 47: 367-78.

[36] Wang B, Fancey KS. Viscoelastically prestressed polymeric matrix composites: An investigation into fibre deformation and prestress mechanisms. Compos Part A Appl Sci Manuf 2018; 111: 106-14.

[37] Perrin F, Bureau MN, Denault J, Dickson JI. Mode I interlaminar crack propagation in continuous glass fiber/polypropylene composites: temperature and molding condition dependence. Compos Sci Technol 2003; 63: 597-607.

[38] Maier C, Calafut T. Polypropylene: the definitive user's guide and databook. William Andrew; 1998. 


\section{CRediT authorship contribution statement}

Bing Wang: Conceptualisation, Methodology, Investigation, Data curation, Formal analysis, Writing - original draft, Project administration, Funding acquisition. Keith A

Seffen: Supervision, Validation, Writing - review \& editing, Funding acquisition.

Simon D Guest: Supervision, Writing - review \& editing, Funding acquisition. Tung-

Lik Lee: Methodology, Resources, Investigation, Writing - review \& editing. Shi

Huang: Investigation, Writing - review \& editing. Shifeng Luo: Investigation, Writing

- review \& editing. Jiawei Mi: Investigation, Writing - review \& editing. 


\section{Graphical Abstract}
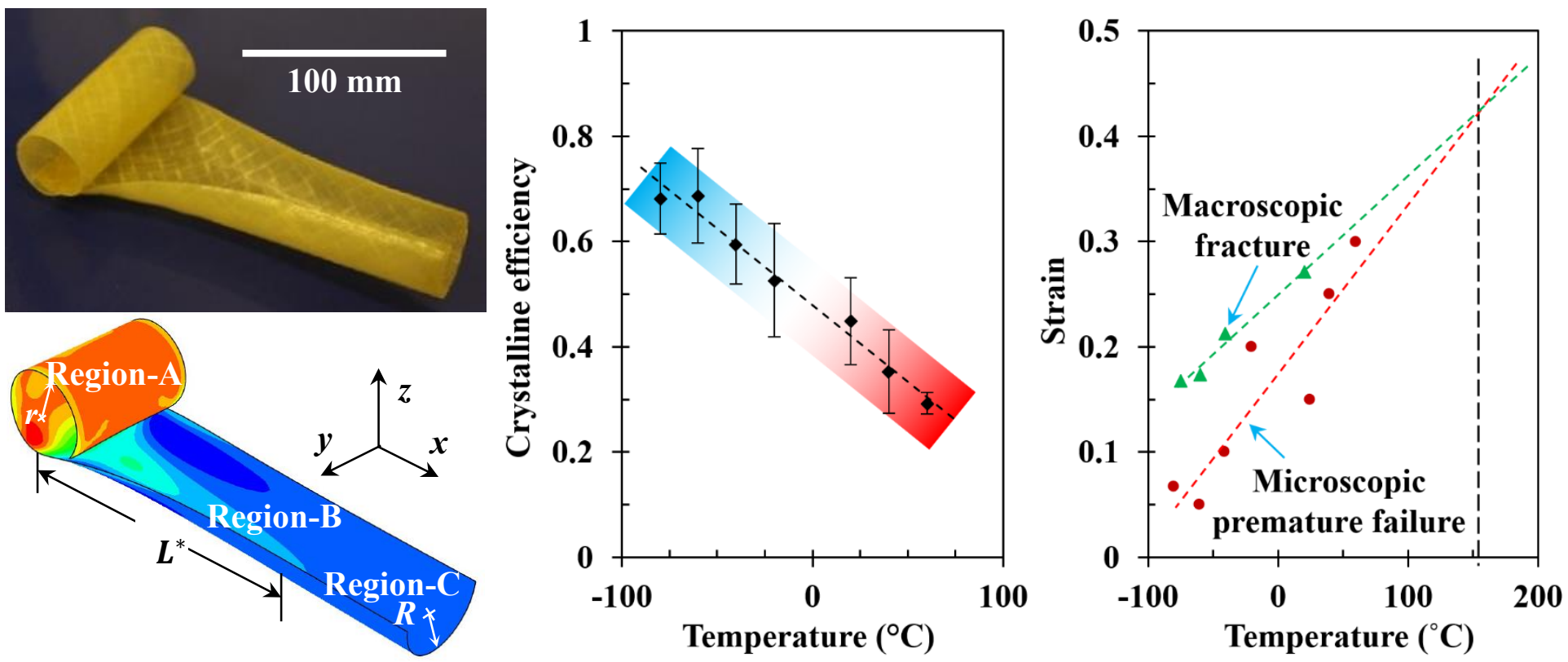